\title{
Homotopy Analysis Method for Nonlinear Periodic Oscillating Equations with Absolute Value Term
}

\author{
Jifeng Cui, Hang Xu, and Zhiliang Lin \\ State Key Laboratory of Ocean Engineering, School of Naval Architecture, Ocean and Civil Engineering, \\ Shanghai Jiao Tong University, Shanghai 200240, China
}

Correspondence should be addressed to Zhiliang Lin; linzhiliang@sjtu.edu.cn

Received 25 March 2015; Revised 23 June 2015; Accepted 5 July 2015

Academic Editor: Raffaele Solimene

Copyright (C) 2015 Jifeng Cui et al. This is an open access article distributed under the Creative Commons Attribution License, which permits unrestricted use, distribution, and reproduction in any medium, provided the original work is properly cited.

Based on the homotopy analysis method (HAM), an analytic approach is proposed for highly nonlinear periodic oscillating equations with absolute value terms. The nonsmoothness of absolute value terms is handled by means of Fourier expansion, and the convergence is accelerated by using the iteration method. Two typical examples which can not be solved by the method of averaging of perturbation technique are employed to illustrate the validity and flexibility of this approach. Rather, accurate approximations are obtained using the HAM-based approach. The proposed approach has general meanings and thus can be used to solve many highly nonlinear periodic oscillating systems with this type of nonsmoothness of absolute value term.

\section{Introduction}

When a periodic oscillating system is governed by a nonlinear differential equation with terms of absolute value, it is hard to gain its analytic approximation of period $T$ by perturbation methods $[1,2]$. This study is important because such kinds of systems are always encountered in the engineering problems. In 1982, the method of averaging is used to study the monitoring system named Lewis regulator by Hagedorn [3]. In 1995, a new asymptotic method is applied to study the large amplitude rolling using only roll extinction data by Chan et al. [4]. In 1999, the main theoretical and experimental aspects connected with the jumps of amplitude of rolling motion in the presence of a bifurcation have been outlined by Francescutto and Contento [5]. All of the above oscillating systems contain the absolute value terms. In this paper, let us consider a type of periodic oscillating systems with absolute value term $[6,7]$

$$
\mathscr{F}[U(t), \dot{U}(t), \ddot{U}(t),|U(t)|]=0,
$$

subject to initial conditions

$$
\begin{aligned}
& U(0)=a, \\
& \dot{U}(0)=0,
\end{aligned}
$$

with $a$ being the amplitude of $U(t), \mathscr{F}$ being a continuous operator, and dot being the differentiation with respect to $t$.

For example, consider the periodic oscillating system governed by

$$
\begin{aligned}
\ddot{U}(t)+\omega_{0}^{2} U(t)+\epsilon|U(t)| U(t) & =0, \\
U(0) & =a, \\
\dot{U}(0) & =0,
\end{aligned}
$$

where $\omega_{0}$ is the natural frequency and $\epsilon$ is a physical parameter. The absolute term $|U(t)|$ brings great difficulty, because the nonlinear term becomes nonsmooth. The method of averaging $[8,9]$ in perturbation theory is frequently used to obtain analytical approximations of (3). With the method of averaging, the 1st-order approximate frequency of (3) reads $[10]$ as follows:

$$
\omega \approx \omega_{0}+\frac{4 \epsilon a}{3 \pi \omega_{0}} .
$$

Unfortunately, the above perturbation result is valid only for small amplitude of oscillation in the case of $\omega_{0}=1$, as shown in Figure 1. It is a common knowledge that in most cases perturbation approximations are valid only for weakly nonlinear 


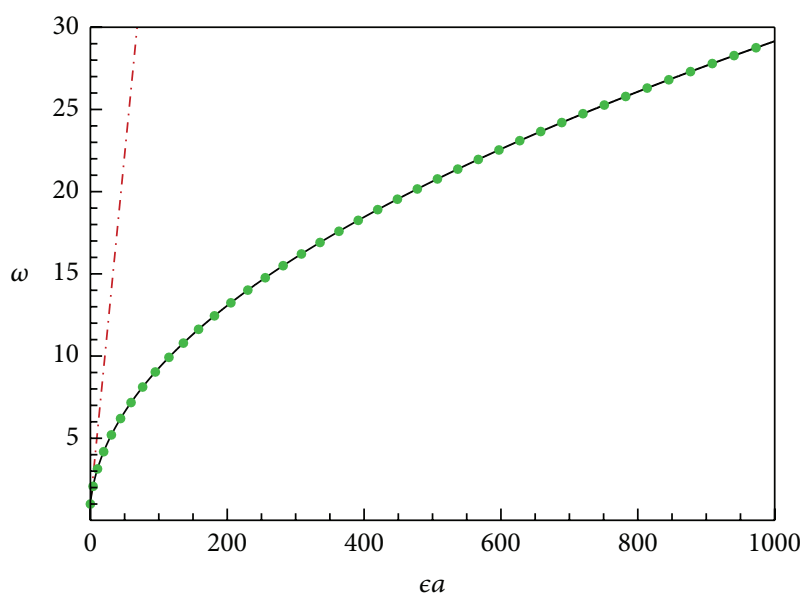

FIGURE 1: Comparison of frequency $\omega$ of (3) given by the 1storder HAM approximation with the exact result and the 1st-order perturbation approximation in the case of $\omega_{0}=1$. Dash-dot: the 1st-order perturbation approximation (4). Filled circle: exact result. Solid line: the 1st-order HAM approximation (5).

problems. To overcome the restrictions of perturbation methods, Liao [11, 12] developed an analytic approximation technique for highly nonlinear problems, namely, the homotopy analysis method (HAM) [13, 14]. The HAM, based on a basic concept in topology, transfers nonlinear problems into an infinite number of linear subproblems which are independent of any small/large physical parameters. So, this technique works efficiently for many nonlinear problems in science and engineering. In particular, the HAM provides a convenient way to guarantee the convergence of solution series. In addition, the HAM provides us with great freedom to choose the equation type of the linear sub problems so that the high-order approximations can be obtained easily in general. Due to these advantages, the HAM has been successfully applied to solve lots of different types of nonlinear problems [15-22]. These successful applications indicate the validity and potential of the HAM.

In many cases, the HAM can give accurate approximations even for problems with high nonlinearity. For example, using the HAM, Liao [11] gave the 1st-order approximation of frequency of (3) in the case of $\omega_{0}=1$ :

$$
\omega \approx \sqrt{1+\frac{8 \epsilon a}{3 \pi}}\left[1-4.96477 \times 10^{-2}\left(\frac{\epsilon a}{\pi+8 \epsilon a / 3}\right)^{2}\right],
$$

which is accurate in the whole interval $0 \leq \epsilon a<+\infty$, as shown in Figure 1. It should be noticed that the 1st-order perturbation result (4) is valid only for small $\epsilon a$, as shown in Figure 1, and the perturbation result (4) given by the method of averaging is invalid for high nonlinearity.

Besides, when $\omega_{0}=0$, the perturbation result (4) has no meaning at all. Thus, when $\omega_{0}=0$, the nonlinearity of (3) is so high that the method of the averaging of perturbation theory does not work.

Recently, based on the HAM combined with Wu's elimination method, a Maple package NOPH is developed by Liu et al. [22] to solve analytic approximations of nonlinear periodic oscillating systems. It is free and available online (http://numericaltank.sjtu.edu.cn/NOPH.htm) with many examples of applications. But this method only works for differentiable nonlinear oscillating systems and can not be used to solve nonlinear periodic oscillating systems like (1).

The current work is an extension of the HAM to solve the nonlinear oscillating systems (1) by means of Fourier expansion and iteration technique.

In this paper, the HAM based on Fourier expansion and iteration will be described in Section 2. Then, the newly developed approach is verified by solving two examples in Section 3 and the validity and the convergence of this approach are also discussed. The conclusions are given in Section 4.

\section{Basic Ideas of the HAM Approach}

2.1. Iteration Approach. By using the sign function

$$
\operatorname{sgn}(U)= \begin{cases}1, & \text { when } U>0, \\ 0, & \text { when } U=0, \\ -1, & \text { when } U<0,\end{cases}
$$

there is

$$
|U|=\operatorname{sgn}(U) U .
$$

Then, (1) can be rewritten as

$$
\mathscr{F}[U(t), \dot{U}(t), \ddot{U}(t), \operatorname{sgn}(U(t)) U(t)]=0 .
$$

Assume that there is a real-valued function set $\left\{U_{i}(t)\right\}_{i=1}^{\infty}$ with the property of uniform convergence; that is, $\lim _{i \rightarrow \infty} U_{i}(t)=$ $U(t)$, and we can construct a family of equations:

$$
\begin{aligned}
\mathscr{F}\left[U_{i}(t), \dot{U}_{i}(t), \ddot{U}_{i}(t), \operatorname{sgn}\left(U_{i-1}(t)\right) U_{i}(t)\right] & =0, \\
i & =1,2,3, \ldots,
\end{aligned}
$$

with

$$
\begin{aligned}
& U_{i}(0)=a, \\
& \dot{U}_{i}(0)=0 .
\end{aligned}
$$

It is obvious that (9) and (10) approach (1) and (2) as $i \rightarrow \infty$.

As soon as an appropriate initial guess of the solution is chosen, the expression of $\operatorname{sgn}\left(U_{i-1}(t)\right)$ is known when solving (9) using the iteration approach. In this way, the problem of (1) and (2) is transformed to (9) and (10). In the subsection, the HAM combined with the Fourier expansion method will be proposed to solve (9) and (10).

2.2. HAM with Fourier Expansion. Assuming that $\left\{U_{0}(t)\right.$, $\left.U_{1}(t), \ldots, U_{i-1}(t)\right\}$ has been obtained, we consider the $i$ th nonlinear subsystem of (9) and (10). Due to considering the periodic solution of the oscillations, the transformation

$$
\begin{aligned}
\tau & =\omega_{i} t, \\
V_{i}(\tau) & =U_{i}(t)
\end{aligned}
$$


is introduced, where $\omega_{i}$ is the frequency of the $U_{i}(t)$. Under the transformation (11), (9) and (10) become

$$
\begin{aligned}
& \mathscr{F}\left[V_{i}(\tau), \omega_{i} \dot{V}_{i}(\tau), \omega_{i}^{2} \ddot{V}_{i}(\tau), \operatorname{sgn}\left(V_{i-1}(\tau)\right) V_{i}(\tau)\right] \\
& \quad=0 \\
& V_{i}(0)=a \\
& \dot{V}_{i}(0)=0
\end{aligned}
$$

where the dot denotes the differentiation with respect to $\tau$.

Note that (12) represents a periodic motion with the period of $2 \pi$. For simplicity, only the cosine-type periodic oscillating systems are considered in this paper. Therefore, $V_{i}(\tau)$ can be expressed by such a set of basis functions

$$
\{\cos (m \tau) \mid m=0,1,2,3, \ldots\} .
$$

That is,

$$
V_{i}(\tau)=\sum_{m=0}^{\infty} \alpha_{m} \cos (m \tau)
$$

where $\alpha_{m}$ are coefficients.

The HAM provides us with great freedom in choosing initial guess of solutions and auxiliary linear operators [11]. Considering the initial conditions (13) and the rule of solution expression (15), the initial guess solution can be chosen as

$$
V_{i, 0}(\tau)=V_{i-1}
$$

Based on the type of the governing equation and the solution expression (15), the auxiliary linear operator is chosen as

$$
\mathscr{L} f=\frac{\partial^{2} f}{\partial \tau^{2}}+f
$$

Note that the operator $\mathscr{L}$ has the property

$$
\mathscr{L}\left(C_{1} \cos \tau+C_{2} \sin \tau\right)=0,
$$

for any constant coefficients $C_{1}$ and $C_{2}$.

According to (12), a nonlinear operator is defined as

$$
\mathcal{N}[f, \omega ; g]=\mathscr{F}\left[f, \omega \frac{\partial f}{\partial \tau}, \omega^{2} \frac{\partial^{2} f}{\partial \tau^{2}}, \operatorname{sgn}(g) f\right] .
$$

The so-called zeroth-order deformation equation is constructed as

$$
\begin{aligned}
& (1-q) \mathscr{L}\left[\Phi_{i}(\tau ; q)-V_{i, 0}(\tau)\right] \\
& \quad=q c_{0} \mathcal{N}\left[\Phi_{i}(\tau ; q), \Omega_{i}(q) ; V_{i-1}(\tau)\right]
\end{aligned}
$$

subject to the initial conditions

$$
\begin{array}{r}
\Phi_{i}(0 ; q)=a, \\
\left.\frac{\partial \Phi_{i}(\tau ; q)}{\partial \tau}\right|_{\tau=0}=0,
\end{array}
$$

where $c_{0}$ is the so-called nonzero convergence-control parameter and $q$ is an embedding parameter.

When $q=0,(20)$ and (21) have the solution

$$
\Phi_{i}(\tau ; 0)=V_{i, 0}(\tau) .
$$

When $q=1,(20)$ and (21) are the same as (12) and (13), respectively, provided

$$
\begin{gathered}
\Phi_{i}(\tau ; 1)=V_{i}(\tau), \\
\Omega_{i}(1)=\omega_{i} .
\end{gathered}
$$

Thus, as the embedding parameter $q$ increases from 0 to 1 , the solution $\Phi_{i}(\tau ; q)$ of the zeroth-order deformation equation deforms from the initial guess $V_{i, 0}(\tau)$ to the exact solution $V_{i}(\tau)$, and so does $\Omega_{i}(q)$ from the initial guess $\omega_{i, 0}$ to the exact frequency $\omega_{i}$, respectively.

From Taylor's theorem, $\Phi_{i}(\tau ; q)$ and $\Omega_{i}(q)$ are expanded in the power series of $q$ as follows:

$$
\begin{aligned}
\Phi_{i}(\tau ; q) & =V_{i, 0}(\tau)+\sum_{n=1}^{\infty} V_{i, n}(\tau) q^{n}, \\
\Omega_{i}(q) & =\omega_{i, 0}+\sum_{n=1}^{\infty} \omega_{i, n} q^{n}
\end{aligned}
$$

where

$$
\begin{aligned}
V_{i, n}(\tau) & =\left.\frac{1}{n !} \frac{\partial^{n} \Phi_{i}(\tau ; q)}{\partial q^{n}}\right|_{q=0}, \\
\omega_{i, n} & =\left.\frac{1}{n !} \frac{d^{n} \Omega_{i}(q)}{d q^{n}}\right|_{q=0}
\end{aligned}
$$

Assume that the value of $c_{0}$ is properly chosen to the point that (24) are convergent at $q=1$. Then, due to (23), one has

$$
\begin{aligned}
V_{i}(\tau) & =V_{i, 0}(\tau)+\sum_{n=1}^{\infty} V_{i, n}(\tau), \\
\omega_{i} & =\omega_{i, 0}+\sum_{n=1}^{\infty} \omega_{i, n} .
\end{aligned}
$$

For the simplicity, two vectors are defined as

$$
\begin{aligned}
& \mathbf{V}_{i, k}=\left\{V_{i, 0}, V_{i, 1}, V_{i, 2}, \ldots, V_{i, k}\right\}, \\
& \boldsymbol{\omega}_{i, k}=\left\{\omega_{i, 0}, \omega_{i, 1}, \omega_{i, 2}, \ldots, \omega_{i, k}\right\} .
\end{aligned}
$$

By differentiating the zeroth-order deformation equations (20) and (21) $m$ times with respect to $q$, then dividing them by $m$ !, and finally setting $q=0$, the so-called $m$ th-order deformation equation is obtained as

$$
\mathscr{L}\left[V_{i, m}(\tau)-\chi_{m} V_{i, m-1}(\tau)\right]=c_{0} \delta_{i, m}(\tau),
$$

subject to the initial conditions

$$
V_{i, m}(0)=V_{i, m}^{\prime}(0)=0
$$


where

$$
\begin{aligned}
& \delta_{i, m}=\frac{1}{(m-1) !} \\
&\left.\frac{\partial^{m-1} \mathcal{N}\left[\Phi_{i}(\tau ; q), \Omega_{i}(q) ; V_{i-1}(\tau)\right]}{\partial q^{m-1}}\right|_{q=0} \\
&=\frac{1}{(m-1) !}\left(\frac{\partial^{m-1}}{\partial q^{m-1}}\right. \\
&\left.. \mathscr{F}\left[\Phi_{i}, \Omega_{i} \frac{\partial \Phi_{i}}{\partial \tau}, \Omega_{i}^{2} \frac{\partial^{2} \Phi_{i}}{\partial \tau^{2}}, \operatorname{sgn}\left(V_{i-1}\right) \Phi_{i}\right]\right)\left.\right|_{q=0}, \\
& \chi_{m}= \begin{cases}0, & m \leq 1, \\
1, & m>1 .\end{cases}
\end{aligned}
$$

Note that $\delta_{i, m}$ is known when the $m$ th-order deformation equation is solved. Therefore, $V_{i, m}(\tau)$ can be obtained by solving the linear equations (28) and (29).

To avoid the difficulty caused by the nondifferentiable function $\operatorname{sgn}\left(V_{i-1}\right)$ in (30), $\delta_{i, m}$ is replaced by the $M$ th-order Fourier series as follows:

$$
\delta_{i, m} \approx \Delta_{i, m}(\tau)=\frac{A_{i, m, 0}}{2}+\sum_{j=1}^{M} A_{i, m, j} \cos (j \tau),
$$

where

$$
A_{i, m, j}=\frac{1}{\pi} \int_{-\pi}^{\pi} \delta_{i, m} \cos (j \tau) d \tau, \quad j=0,1,2, \ldots
$$

Then, the general solution of (28) reads

$$
\begin{aligned}
V_{i, m}(\tau) \approx & \chi_{m} V_{i, m-1}(\tau)+V_{i, m}^{*}(\tau)+C_{i, 1} \cos \tau \\
& +C_{i, 2} \sin \tau,
\end{aligned}
$$

where

$$
V_{i, m}^{*}(\tau)=\mathscr{L}^{-1}\left[c_{0} \Delta_{i, m-1}(\tau)\right],
$$

and the integral coefficients

$$
\begin{aligned}
& C_{i, 1}=-\chi_{m} V_{i, m-1}(0)-V_{i, m}^{*}(0), \\
& C_{i, 2}=0,
\end{aligned}
$$

are determined by the initial condition (29).

Note that $\omega_{i, m-1}$ is unknown so far. So, one additional algebraic equation is needed to determine $\omega_{i, m-1}$. Equation (28) can be rewritten as

$$
\mathscr{L}\left[V_{i, m}(\tau)-\chi_{m} V_{i, m-1}(\tau)\right]=c_{0} \sum_{j=0}^{M} A_{i, m-1, j} \cos (j \tau) .
$$

According to the linear auxiliary operator (17), if $A_{i, m-1,1} \neq$ $0, V_{i, m}(\tau)$ contains the so-called secular term $\tau \sin (\tau)$, which is not periodic. Such kind of secular terms must be avoided. Thus, $A_{i, m-1,1}=0$ is enforced, which provides an additional algebraic equation for $\omega_{i, m-1}$. At this point, $\omega_{i, 0}, \omega_{i, 1}, \ldots, \omega_{i, m-1}$ can be obtained in sequence. In this way, the linear deformation equations (28) and (29) can be successively solved.

The $K$ th-order approximations of $V_{i}(\tau)$ and $\omega_{i}$ are defined as

$$
\begin{aligned}
& V_{i} \approx \sum_{m=0}^{K} V_{i, m}(\tau), \\
& \omega_{i} \approx \sum_{m=0}^{K} \omega_{i, m},
\end{aligned}
$$

respectively. Obviously, the Kth-order homotopy-approximation of $V_{i}(\tau)$ satisfies the initial condition (13). It is usually much better than the initial approximation $V_{i, 0}(\tau)$, if the convergence-control $c_{0}$ is properly chosen. In order to choose a proper value of $c_{0}$, the squared residual error is defined as follows:

$$
\mathscr{E}_{i}=\int_{0}^{2 \pi}\left(\mathcal{N}\left[V_{i}(\tau), \omega_{i} ; V_{i-1}(\tau)\right]\right)^{2} d \tau .
$$

For the sake of computational efficiency, the squared residual error $\mathscr{E}_{i}$ is calculated numerically

$$
\begin{aligned}
\mathscr{E}_{i} & \approx \overline{\mathscr{E}}_{i}\left(c_{0}\right) \\
& =\frac{1}{N+1} \sum_{k=0}^{N}\left(\left.\mathcal{N}\left[V_{i}(\tau), \omega_{i} ; V_{i-1}(\tau)\right]\right|_{\tau=k \Delta \tau}\right)^{2},
\end{aligned}
$$

where $\Delta \tau=2 \pi / N$ and $N$ is an integer. $N=50$ is set in this paper. Given the initial guess $V_{i, 0}(\tau)$ and the auxiliary linear operator $\mathscr{L}$, the discrete squared residual error $\overline{\mathscr{E}}_{i}$ is only dependent on the convergence-control parameter $c_{0}$, whose optimal value is determined by the minimum of $\overline{\mathscr{E}}_{i}$. Unlike other analytical methods, the HAM provides a convenient way to guarantee the convergence of the series solution $[22$, 23].

\section{Examples}

In this section, the validity of the proposed iteration approach is illustrated by two examples.

Example 1. First, consider the following equation:

$$
\ddot{U}(t)+\epsilon|U(t)| U(t)=0 .
$$

According to (11) and (12), (42) becomes

$$
\omega_{i}^{2} \ddot{V}_{i}(\tau)+\epsilon \operatorname{sgn}\left(V_{i-1}(\tau)\right) V_{i}^{2}(\tau)=0 .
$$

And the first initial guess of the solution is given as follows:

$$
V_{1,0}(\tau)=V_{0}(\tau)=a \cos \tau .
$$


TABLE 1: Comparison of $\omega_{1,0}$ and $\omega_{1,1}$ with $\omega_{N}$ under different amplitude $a$ in the case of $\epsilon=1$.

\begin{tabular}{lccccc}
\hline$a$ & $\omega_{1,0}$ & $\omega_{1,1}$ & $\omega_{N}$ & $R_{0}$ & $R_{1}$ \\
\hline 1 & 0.92132 & 0.91464 & 0.91468 & $0.73 \%$ & $0.05 \%$ o \\
10 & 2.91346 & 2.89198 & 2.89247 & $0.73 \%$ & $0.17 \%$ o \\
100 & 9.21318 & 9.15655 & 9.14681 & $0.73 \%$ & $0.38 \%$ o \\
1000 & 29.1346 & 28.9556 & 28.9247 & $0.73 \%$ & $1.06 \%$ o \\
\hline
\end{tabular}

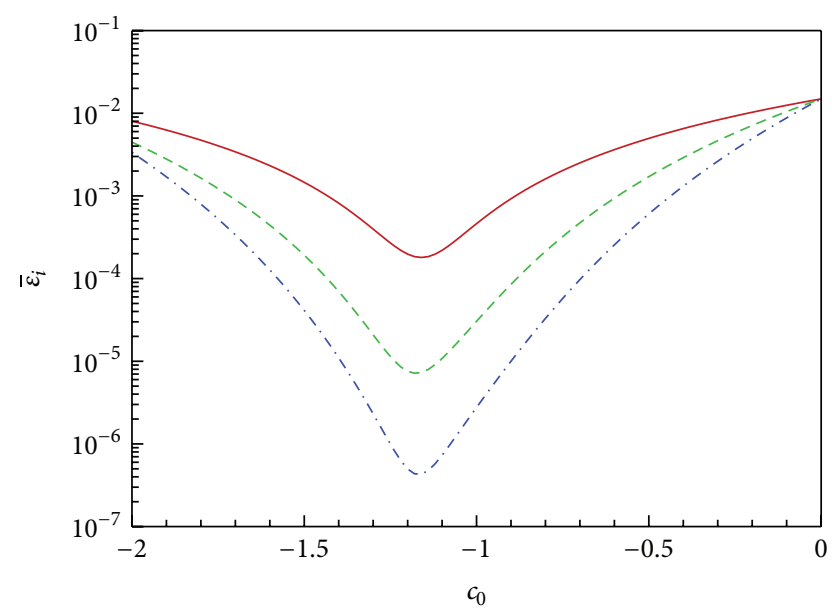

Figure 2: Discrete squared residual $\overline{\mathscr{E}}_{i}\left(c_{0}\right)$ in the case of $\epsilon=1$, $a=1$. Solid line: 1 st-order approximation. Dashed line: 2 nd-order approximation. Dash-dot line: 3rd-order approximation.
The term $\delta_{i, m}$ in (28) becomes

$$
\begin{aligned}
\delta_{i, m}= & \sum_{l=0}^{m-1} \ddot{V}_{i, l}\left(\sum_{j=0}^{m-1-l} \omega_{i, j} \omega_{i, m-1-l-j}\right) \\
& +\epsilon \operatorname{sgn}\left(V_{i-1}\right) \sum_{j=0}^{m-1} V_{i, j} V_{i, m-1-j} .
\end{aligned}
$$

By using computer algebra software Mathematica, approximate solutions of the high-order deformation equations can be obtained.

When $i=1$, the 1st-order and 2nd-order approximations of frequency $\omega$ can be sequentially obtained:

$$
\begin{aligned}
& \omega_{1,0}=\sqrt{\frac{8 \epsilon a}{3 \pi}}, \\
& \omega_{1,1}=\sqrt{\frac{8 \epsilon a}{3 \pi}}+\frac{313765789237 \sqrt{2 / 3} a^{3 / 2} \epsilon^{3 / 2} c_{0}}{8467558748649 \pi^{3 / 2}},
\end{aligned}
$$

where

$$
c_{0}(a)= \begin{cases}-1.16152+1.05313 \ln a-0.369972 \ln ^{2} a+0.0474005 \ln ^{3} a, & (0<a<30), \\ -0.285884+0.109898 \ln a-0.0109417 \ln ^{2} a, & (30 \leq a \leq 100), \\ -0.210627+0.0884892 \ln a-0.0126579 \ln ^{2} a+0.000613544 \ln ^{3} a, & (100 \leq a \leq 1000) .\end{cases}
$$

Here, some supplemental statements are needed to elaborate the origin of the piecewise function $c_{0}(a)$. Figure 2 illustrates that the optimal convergence-control parameter $c_{0}$ can be chosen as $-117 / 100$ by plotting residual curves in the case of $a=1, \epsilon=1$. Similarly, in the case of $\epsilon=1$, the following data set

$$
\begin{aligned}
& \left\{\left(a, c_{0}\right) \mid\left(1,-\frac{117}{100}\right),\left(2,-\frac{29}{50}\right),\left(3,-\frac{19}{50}\right), \ldots,\right. \\
& \left.\left(1000,-\frac{11}{10000}\right)\right\}
\end{aligned}
$$

can be obtained. Further, the piecewise function $c_{0}(a)$ can be obtained easily by using the Mathematica built-in function Fit. Then, $R_{i}=\left|\left(\omega_{1, i}-\omega_{N}\right) / \omega_{N}\right|(i=0,1)$ is defined to measure the relative error between $\omega_{1, i}$, obtained by HAM, and the numerical frequency $\omega_{N}$, listed in Table 1 , which shows that $\omega_{1,1}$ is valid even with a large amplitude such as $a=1000$. Both Table 1 and Figure 3 verify the effectiveness of the frequency obtained by HAM. The optimal convergencecontrol parameter $c_{0}=-119 / 100$ is chosen according to the Mathematica built-in function NMinimize $\left[\overline{\mathscr{E}}_{1}, c_{0}\right]$ in the case of $\epsilon=a=1$. Without loss of generality, let $K=3$, that is, a 3rd-order homotopy-iteration approach applied. Thus, the solutions $V_{1,1}(\tau), V_{1,2}(\tau), \ldots, V_{1, m-1}(\tau)$ are obtained by the initial guess of the solution $V_{1,0}(\tau)=$ $V_{0}=a \cos (\tau)$. Next, the initial guess of the solution will be updated by $V_{1}=\sum_{m=0}^{K} V_{1, m}(\tau)$ and a range of new solutions $V_{2,1}(\tau), V_{2,2}(\tau), \ldots, V_{2, m-1}(\tau)$ will be obtained again, which can be called $K$ th-order and one iteration. If we loop $s$ times, this process will be called $K$ th-order and $s$ times iteration. Besides, let $M=20$, and $\delta_{s, m}$ is approximated by the first 20 lower-frequency terms only. The homotopyapproximation at each iteration, the corresponding discrete square residual, and the homotopy-approximations of frequency $\omega_{s}$ are listed in Table 2 . Table 2 shows that the square residual of the homotopy-approximation decreases to $10^{-8}$ and the frequency $\omega$ of the oscillating system (42) converges to the value 0.9146812713 as the iteration increases. In Figure 4, the homotopy-approximation of $U(t)$ after the 10 times iteration is compared with the numerical result. It shows that the homotopy-approximation and the numerical result agree very well. 
TABle 2: The discrete square residual $\overline{\mathscr{E}}_{s}$ and the homotopyapproximations of frequency $\omega_{s}$ in the case of $\epsilon=a=1$ by the 3rd-order homotopy-iteration approach with normal initial approximation $V_{0}(\tau)=a \cos (\tau)$ and the optimal convergencecontrol parameter $c_{0}=-(119 / 100)$.

\begin{tabular}{lcc}
\hline$s$, iteration times & $\overline{\mathscr{E}}_{s}$ & $\omega_{s}$ \\
\hline 1 & $1.844177938 \times 10^{-4}$ & 0.9147546341 \\
2 & $4.350266713 \times 10^{-7}$ & 0.9146813012 \\
3 & $9.821549663 \times 10^{-8}$ & 0.9146812713 \\
4 & $9.680249431 \times 10^{-8}$ & 0.9146812713 \\
5 & $9.670548211 \times 10^{-8}$ & 0.9146812713 \\
6 & $9.653542104 \times 10^{-8}$ & 0.9146812713 \\
\hline
\end{tabular}

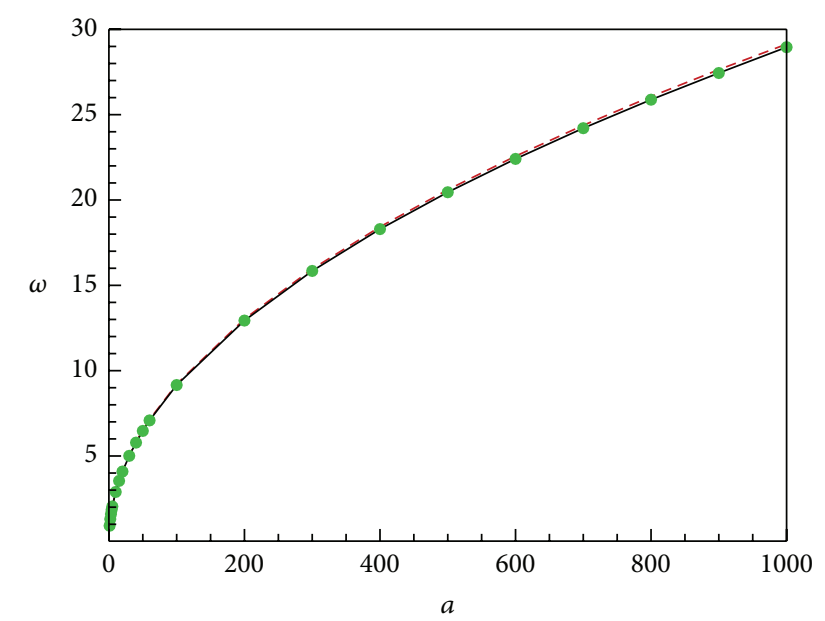

FIgURE 3: Comparison of the $\omega_{1,0}$ and $\omega_{1,1}$ with $\omega_{N}$ in the case of $\epsilon=1$. Dashed line: $\omega_{1,0}$. Solid line: $\omega_{1,1}$. Filled circle: $\omega_{N}$.

Example 2. Furthermore, consider

$$
\ddot{U}(t)+\epsilon\left(|U(t)|+U^{3}(t)\right)=0 .
$$

According to (11) and (12), (49) becomes

$$
\omega_{i}^{2} V_{i}^{\prime \prime}(\tau)+\epsilon \operatorname{sgn}\left(V_{i-1}(\tau)\right) V_{i}(\tau)+\epsilon V_{i}^{3}(\tau)=0 .
$$

The solving procedures are the same as those defined in Section 2, except that the term $\delta_{i, m}$ in (28) becomes

$$
\begin{aligned}
\delta_{i, m}= & \sum_{l=0}^{m-1} V_{i, l}^{\prime \prime}\left(\sum_{j=0}^{m-1-l} \omega_{i, j} \omega_{i, m-1-l-j}\right) \\
& +\epsilon \operatorname{sgn}\left(V_{i-1}\right) V_{i, m-1} \\
& +\epsilon \sum_{l=0}^{m-1} V_{i, l}\left(\sum_{j=0}^{m-1-l} V_{i, j} V_{i, m-1-l-j}\right) .
\end{aligned}
$$

The basic idea of choosing the suitable values of parameters $\epsilon=a=1, M=20, K=3$, and $c_{0}=-4 / 25$ and computing the approximations at the first several orders by using Mathematica software is similar to Example 1. The homotopyapproximation at each iteration, the corresponding discrete
TABLE 3: The discrete square residual $\overline{\mathscr{E}}_{s}$ and the homotopyapproximations of frequency $\omega_{s}$ in the case of $\epsilon=a=1$ by the 3 rd-order homotopy-iteration approach with normal initial approximation $V_{0}(\tau)=a \cos (\tau)$ and the optimal convergencecontrol parameter $c_{0}=-(4 / 25)$.

\begin{tabular}{lcc}
\hline$s$, iteration times & $\overline{\mathscr{E}}_{s}$ & $\omega_{s}$ \\
\hline 1 & $2.056111269 \times 10^{-1}$ & 0.88960 \\
3 & $4.583588352 \times 10^{-2}$ & 1.16473 \\
5 & $3.666051972 \times 10^{-3}$ & 1.16042 \\
7 & $4.616399200 \times 10^{-4}$ & 1.15628 \\
8 & $1.927268621 \times 10^{-4}$ & 1.15614 \\
9 & $9.925323843 \times 10^{-5}$ & 1.15614 \\
\hline
\end{tabular}

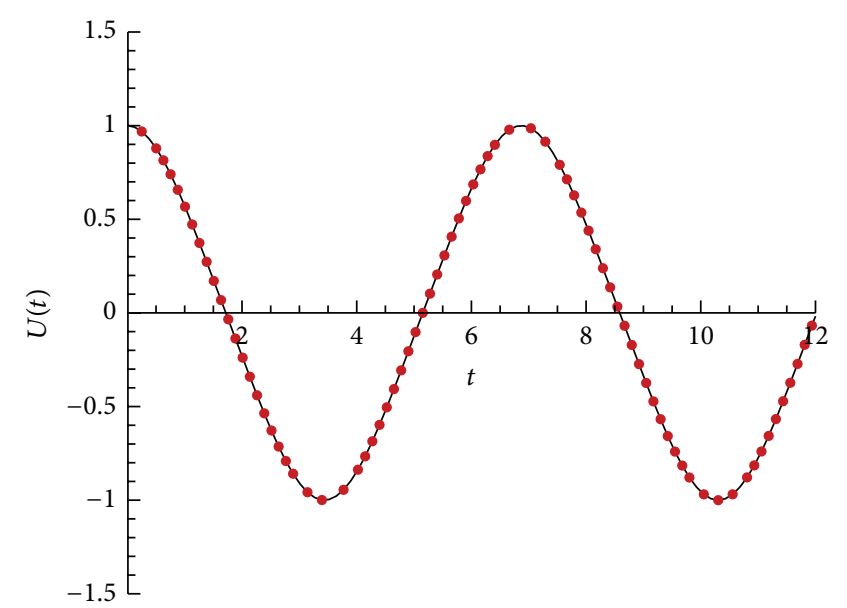

FIGURE 4: Comparison of the homotopy-approximation of $U(t)$ by the 3rd-order and 10 times iteration with normal initial approximation $V_{0}(\tau)=a \cos (\tau)$ in the case of $\epsilon=a=1$ by means of $c_{0}=$ $-119 / 100$ with numerical results. Solid line: 3rd-order homotopyiteration approach. Filled circle: numerical result.

square residual, and the homotopy-approximations of frequency $\omega_{s}$ are listed in Table 3 . It shows that the discrete square residual of the homotopy-approximation decreases to $10^{-5}$ and the frequency $\omega$ of the oscillating system (49) converges to the value 1.15614 as the iteration times $s$ increases to 8 . This illustrates that the frequency $\omega$ of the nonlinear oscillating system (49) can be well approximated. As shown in Figure 5, the equilibrium point of the nonlinear oscillating system deviates from 0 , which causes the slow convergence of the homotopy-series solution. The homotopyapproximation of $U(t)$ by the 3rd-order and 10 times iteration is compared with the numerical result in Figure 5. It shows that the homotopy-approximation and the numerical result are in well agreement.

\section{Conclusions}

Based on the homotopy analysis method, an analytic approach is proposed for highly nonlinear periodic oscillating equations containing absolute value terms. The nonsmoothness of absolute value terms is handled by means 


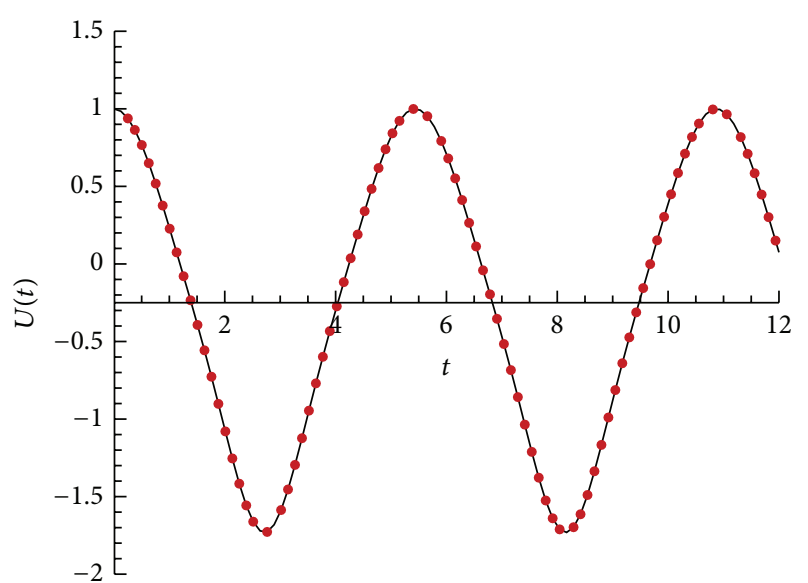

FIGURE 5: Comparison of the homotopy-approximation of $U(t)$ by the 3rd-order and 10 times iteration with normal initial approximation $V_{0}(\tau)=a \cos (\tau)$ in the case of $\epsilon=a=1$ by means of $c_{0}=-4 / 25$ with numerical results. Solid line: 3 rd-order homotopyiteration approach. Filled circle: numerical result.

of Fourier expansion, and the convergence is accelerated by means of iteration approach. Two typical examples are employed to illustrate the validity and flexibility of this approach. Note that they can not be solved by means of the method of averaging in perturbation theory. Rather, accurate approximations are obtained using the HAM-based approach proposed in this paper.

This approach has general meanings and thus can be used to solve many highly nonlinear periodic oscillating systems with this type of nonsmoothness in science and engineering.

\section{Conflict of Interests}

The authors declare that there is no conflict of interests regarding the publication of this paper.

\section{Acknowledgments}

The authors would like to express their sincere thanks to Professor Liqun Chen and Mr. Jing Li for their helpful and enlightening discussions. They are grateful to the National Natural Science Foundation of China (Approval nos. 11272209 and 51209136) for the financial support.

\section{References}

[1] H. Richard, It's a Nonlinear World, Springer, New York, NY, USA, 2011.

[2] J. P. D. Hartog, "Forced vibration with coulomb and viscous damping," Transactions of the American Society of Mechanical Engineers, vol. 53, pp. 107-115, 1931.

[3] P. Hagedorn, Non-Linear Oscillations, Clarendon Press, Oxford, UK, 1982.

[4] H. S. Y. Chan, Z. Xu, and W. L. Huang, "Estimation of nonlinear damping coefficients from large-amplitude ship rolling motions," Applied Ocean Research, vol. 17, no. 4, pp. 211-224, 1995.
[5] A. Francescutto and G. Contento, "Bifurcations in ship rolling: experimental results and parameter identification technique," Ocean Engineering, vol. 26, no. 11, pp. 1095-1123, 1999.

[6] A. H. Nayfeh and D. T. Mook, Nonlinear Oscillations, John Wiley \& Sons, New York, NY, USA, 1979.

[7] C. Hayashi, Nonlinear Oscillations in Physical Systems, McGrawHill, New York, NY, USA, 1964.

[8] J. A. Sanders and F. Verhulst, Averaging Methods in Nonlinear Dynamical Systems, Springer, New York, NY, USA, 1985.

[9] D. Graham and D. McRuer, Oscillations in Nonlinear Control Systems, Dover, New York, NY, USA, 1971.

[10] Y. Z. Liu and L. Q. Chen, Nonlinear Vibrations, Higher Education Press, Beijing, China, 2001 (Chinese).

[11] S. J. Liao, Beyond Perturbation: Introduction to the Homotopy Analysis Method, Chapman \& Hall, CRC Press, Boca Raton, Fla, USA, 2003

[12] S. J. Liao, Homotopy Analysis Method in Nonlinear Differential Equations, Springer, New York, NY, USA, 2011.

[13] S. J. Liao and Y. Tan, "A general approach to obtain series solutions of nonlinear differential equations," Studies in Applied Mathematics, vol. 119, no. 4, pp. 297-354, 2007.

[14] S. J. Liao, "An optimal homotopy-analysis approach for strongly nonlinear differential equations," Communications in Nonlinear Science and Numerical Simulation, vol. 15, no. 8, pp. 2003-2016, 2010.

[15] T. Hayat and M. Sajid, "On analytic solution for thin film flow of a fourth grade fluid down a vertical cylinder," Physics Letters A, vol. 361, no. 4-5, pp. 316-322, 2007.

[16] S. Abbasbandy, "Solitary wave solutions to the KuramotoSivashinsky equation by means of the homotopy analysis method," Nonlinear Dynamics, vol. 52, no. 1-2, pp. 35-40, 2008.

[17] F. M. Allan, "Construction of analytic solution to chaotic dynamical systems using the Homotopy analysis method," Chaos, Solitons and Fractals, vol. 39, no. 4, pp. 1744-1752, 2009.

[18] M. Turkyilmazoglu, "Purely analytic solutions of the compressible boundary layer flow due to a porous rotating disk with heat transfer," Physics of Fluids, vol. 21, no. 10, Article ID 106104, 2009.

[19] S. X. Liang and D. J. Jeffrey, "An efficient analytical approach for solving fourth order boundary value problems," Computer Physics Communications, vol. 180, no. 11, pp. 2034-2040, 2009.

[20] V. Kuppalapalle and R. A. Gorder, Nonlinear Flow Phenomena and Homotopy Analysis: Fluid Flow and Heat Transfer, Springer, New York, NY, USA, 2013.

[21] J. Cheng, S.-P. Zhu, and S.-J. Liao, "An explicit series approximation to the optimal exercise boundary of American put options," Communications in Nonlinear Science and Numerical Simulation, vol. 15, no. 5, pp. 1148-1158, 2010.

[22] Y. P. Liu, S. J. Liao, and Z. B. Li, "Symbolic computation of strongly nonlinear periodic oscillations," Journal of Symbolic Computation, vol. 55, pp. 72-95, 2013.

[23] S. J. Liao, Advances in the Homotopy Analysis Method, World Scientific, New York, NY, USA, 2013. 


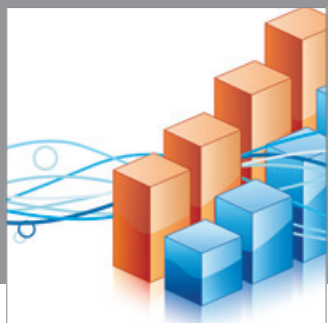

Advances in

Operations Research

mansans

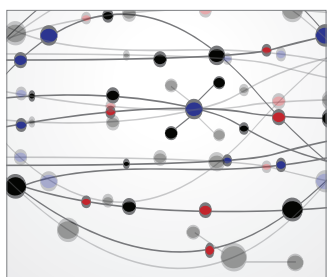

The Scientific World Journal
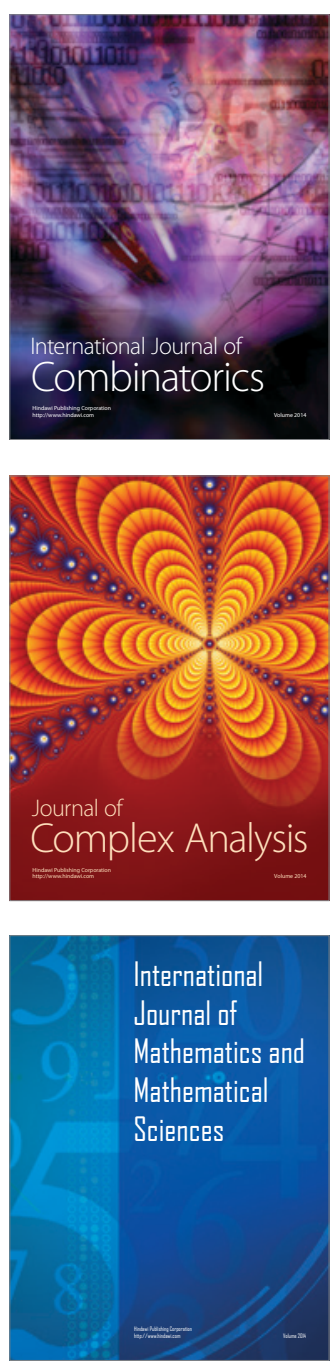
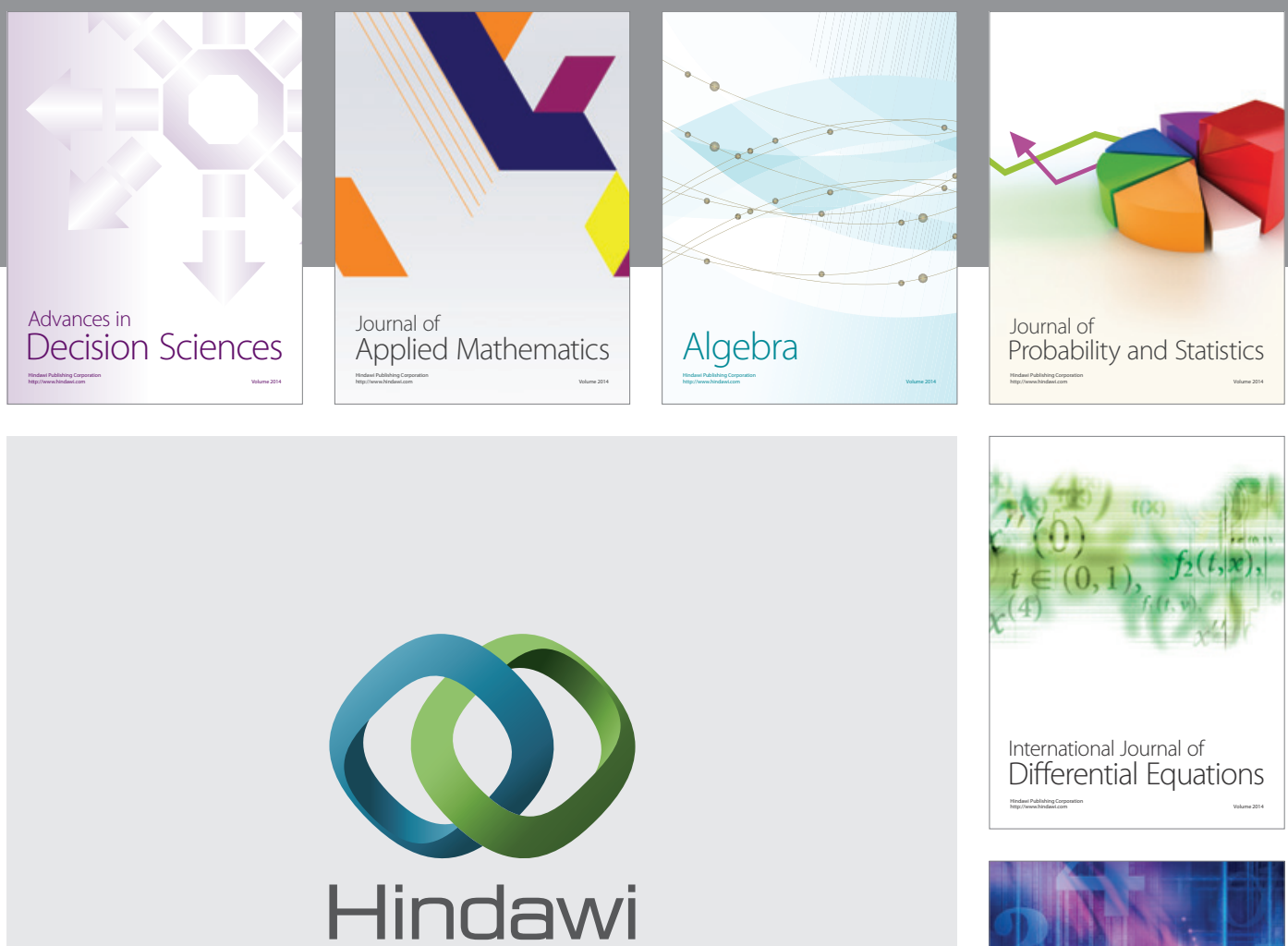

Submit your manuscripts at http://www.hindawi.com
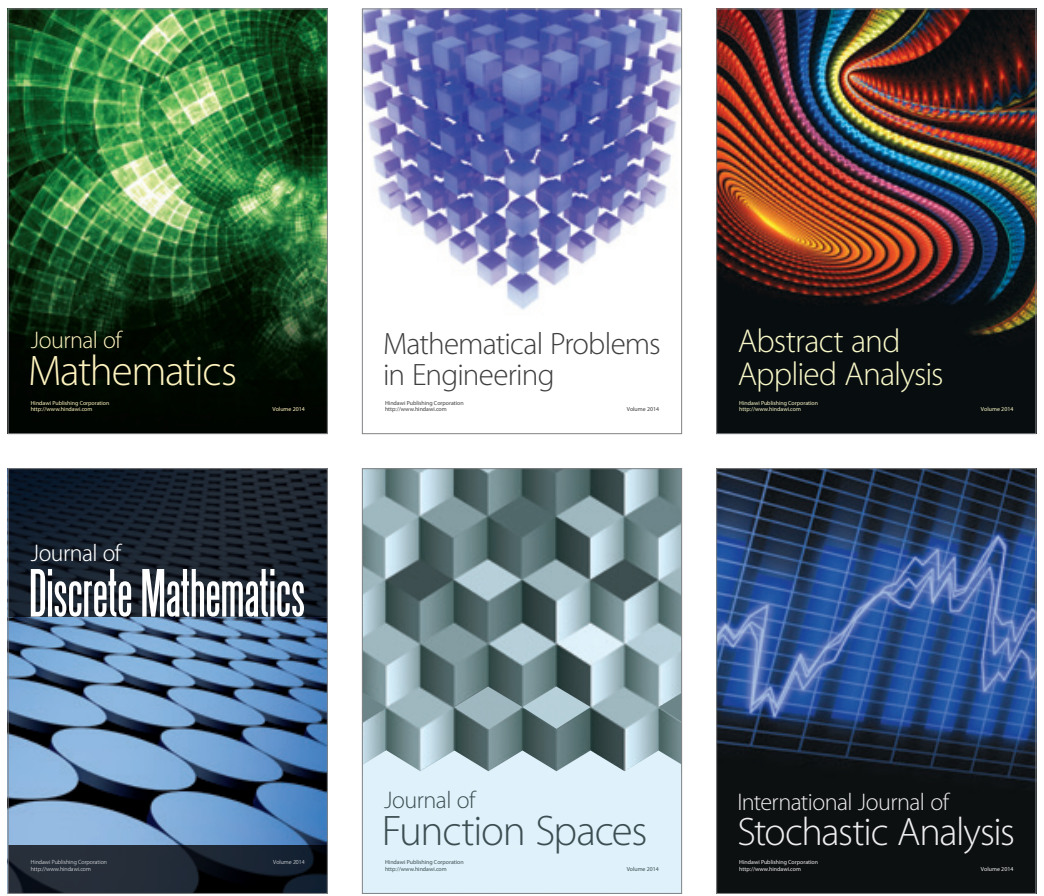

Journal of

Function Spaces

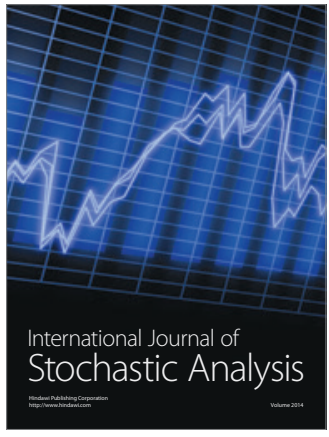

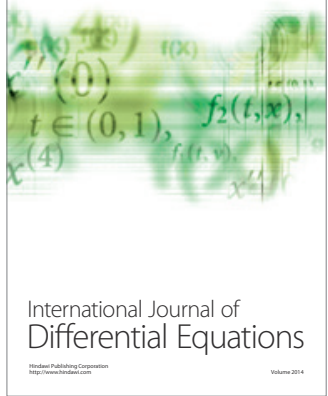
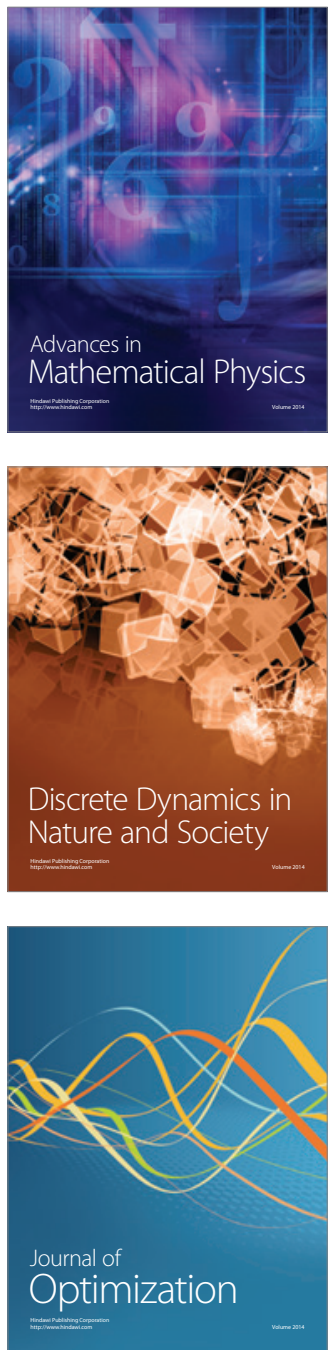